http://jmscr.igmpublication.org/home/ ISSN (e)-2347-176x ISSN (p) 2455-0450 crossref DOI: https://dx.doi.org/10.18535/jmscr/v9i9.10

\footnotetext{
Dournal Of Medical Science And Clinical Research

IGM Publication

An Official Publication of IGM Publication
}

\title{
Tyrosinemia Type 1 Presenting with Congestive Cardiac Failure
}

\author{
Authors \\ Dr Snehal Sonune ${ }^{1}$, Dr Punam Uke ${ }^{2}$, Dr Ankita Kamble ${ }^{3}$, Dr Akshay Wanvat ${ }^{4}$, \\ Dr Poonam Mane ${ }^{5}$, Dr Shubham Meshram ${ }^{6}$ \\ 1,3,4,5,6 Resident of MD Pediatrics, Grant Medical College and JJ Hospital Mumbai \\ ${ }^{2}$ Assistant Professor Department of Pediatrics Grant Medical College and JJ Hospital Mumbai
}

\section{Introduction}

Tyrosinemia is an autosomal recessive inherited condition characterized by inability of cells to process tyrosine. It is very rare cause of chronic liver diseases in childhood and usually begins in infants less than 6 months of age. However some may present in later childhood. We present a case of Tyrosinemia type 1 who was presented with Congestive Cardiac Failure and was managed successfully.

\section{Case Report}

10 months old female child, 2nd Birth by order, Born of consanguineous marriage (3rd degree) was brought by parents in casualty with c/o Weight Loss since 2 months, on and off fever since 2 months, acute gastroenteritis since 8 days, Ascites since 8 days, Respiratory Distress since 2 days. Child had no history of admission in the past.child had yellow coloured stool and yellow coloured urine with offensive smell. Birth history was normal without any postnatal complications. Child was immunised up to 14 week's (partially immunised). H/o cow milk + . Child had a delayed developmental milestones. On Physical examination child was irritable, pale Distended Abdomen, afebrile, peripheral pulses well felt,
HR- 154/min,RR-58/ min, SCR, ICR+, BP90/60 mmHg in right upper arm in supine position. Weight was $5.2 \mathrm{~kg}$ Height- $65 \mathrm{~cm} \mathrm{Z}$ score is between <-3 SD. Child had no signs of fat soluble vitamin deficiency. No scratch marks seen. No clubbing, no cyanosis seen. Liver was enlarged with span of $8 \mathrm{~cm}$ in midclavicular line Non tender, firm consistency. The spleen was 1-2 cm palpable. Minimal Ascites +. The infant was irritable. No signs of meningeal irrigation. Laboratory investigations was done s/o following values CBC HB-6.7, TLC-9,090, Platelet-2.55 lac. Sr potassium- 3.4, sr sodium- 142, LFT SGOT 74, SGPT-34, Total bilirubin- 0.42 , direct/ indirect0.19/0.23, Alpo4-831, Albumin- 2.8, PT-25.6, INR-1.97.GGT-96, RFT-WNL. RBS-70, serum NH 3-97. Peripheral smear s/o microcytic +hypochromic+ cells. Iron studies s/o Iron deficiency Anemia.AFP levels Raised.(44500). Child kept NBM IVM on 02 by prongs. and started on Inj Taxim, Inj Metro and other symptomatic management USG abdo s/o Borderline Hepatomegaly with Altered echo texture s/o Liver Parenchymal Disease. B/L kidneys appear Bulky in size with marginally raised cortical echogenecity and maintained cortico-medullary demarcation s/o Acute kidney 
Injury. Minimal Ascites. Vit $\mathrm{k}$ and 2 FFP transfusion given due to deranged coagulation profile. $2 \mathrm{PCV}$ transfusions was given ivo CCF. Severe Acute Malnutrition management added. ivo<-3SD score. After PCV transfusions CCF settled so 02 tapered and omitted child orally allowed. BCS came negative so antibiotics omitted after 10 days. TMS and Urine GCMS was sent s/o Tyrosinemia type 1.BERA-WNL, TFTWNL. Pediatric gastroenterologist opinion was taken advised to send genetic work up (Whole Exom Sequencing). Counselling for Liver transplant was done. Genetic work up was sent report a waited. The above findings and Lab Data s/o Tyrosinemia type 1.

Child was discharged on Severe Acute Malnutrition management. Dietary counselling done parents were counselled on the need for liver transplantation and importance of ANC testing in future pregnancy.

\section{Discussion}

The common diffential diagnosis for such presentation are extra hepatic Obstruction, Metabolic disorder, infective pathology, viral Markers to rule out the infective cause.USG rule out the extrahepatic billiary Obstruction. Thyroid disorder was ruled out. Due to H/o severe weight loss with offensive smell of urine. We had planned to rule out Metabolic disorder by sending TMS (Tandem Mass Spectrometry) and Urinary GCMS (Gas Chromatography Mass Spectrometry) s/o Tyrosinemia type 1 .

Tyrosinemia type 1 is rare Autosomal recessive genetic metabolic disorder characterized by lack of enzyme fumarylacetone Hydrolase (FAH) which is needed for the final breakdown of amino acid tyrosine. Failure to properly break down tyrosine and it's metabolites in liver, potentially resulting in severe liver disease. Tyrosine may also accumulate in the kidneys and central nervous system. The affected infant appears normal at birth and typically presents between 2-6 months of age but rarely may become symptomatic in the 1st month bor appear healthy beyond 1 st year of life. The earlier the presentation the poorer the prognosis. In Tyrosinemia type 2 Liver and kidney function are normal as serum concentration of other amino acid and succinylacetone. Tyrosinemia type 2 is caused by TAT gene mutations. Tyrosinemia type 3 caused by deficiency of 4 hydroxy phenylpuruvate dioxygenase

Treatment includes nutritional support $b$ (Adequate calories tyrosine, phenylalanine free diet) and Nitisinone. Ultimately liver transplantation is needed in most of the patients as they develop cirrhosis or hepatocellular carcinoma. Monitoring recommended from infancy.

\section{Acknowledgment}

We would like to thank Dr Bela Verma ma'am Head of Department of pediatrics, to allow us to publish this manuscript.

\section{References}

1. Morrow G, Tanguay RM. Biochemical and Clinical Aspects of Hereditary Tyrosinemia Type 1. Adv Exp Med Biol. 2017,959:9-21. doi: 10.1007/978-3-31955780-9 2. PMID: 28755181.

2. van Ginkel WG, Rodenburg IL, Harding CO, Hollak CEM, Heiner-Fokkema MR, van Spronsen FJ. Long-Term Outcomes and Practical Considerations in the Pharmacological Management of Tyrosinemia Type 1. Paediatr Drugs. 2019 Dec;21(6):413-426. doi: 10.1007/s40272019-00364 4. PMID: 31667718; PMCID: PMC6885500.

3. Mirani S, Poojari V, Shetty NS, Shah I. Outcome of Tyrosinemia Type 1 in Indian Children. J Clin Exp Hepatol. 2021 JanFeb;11(1):9-13. doi: 10.1016/j.jceh.2020.07.002. Epub $2020 \mathrm{Jul}$ 11. PMID: 33679043; PMCID: PMC7897851.

4. Chinsky JM, Singh R, Ficicioglu C, van Karnebeek CDM, Grompe M, Mitchell G, 
Waisbren SE, Gucsavas-Calikoglu M, Wasserstein MP, Coakley K, Scott CR. Diagnosis and treatment of tyrosinemia type I: a US and Canadian consensus group review and recommendations. Genet Med. $2017 \quad$ Dec;19(12). doi: 10.1038/gim.2017.101. Epub 2017 Aug 3. PMID: 28771246; PMCID: PMC5729346. 